\title{
Improved HaloTag Ligand Enables BRET Imaging With NanoLuc
}

\author{
Ovia Margaret Thirukkumaran ${ }^{1,2}$, Congrong Wang ${ }^{1}$, Nnamdi Joseph Asouzu ${ }^{1}$, \\ Eduard Fron ${ }^{2}$, Susana Rocha ${ }^{2}$, Johan Hofkens ${ }^{2}$, Luke D. Lavis ${ }^{3}$ and Hideaki Mizuno ${ }^{1 *}$ \\ ${ }^{1}$ Laboratory for Biomolecular Network Dynamics, Biochemistry, Molecular and Structural Biology Section, Department of \\ Chemistry, KU Leuven, Heverlee, Belgium, ${ }^{2}$ Chem Tech-Molecular Imaging and Photonics, Department of Chemistry, KU \\ Leuven, Heverlee, Belgium, ${ }^{3}$ Howard Hughes Medical Institute, Ashburn, VA, United States
}

Bioluminescence resonance energy transfer (BRET) from an exceptionally bright luciferase, NanoLuc, to a fluorescent HaloTag ligand is gaining momentum to monitor molecular interactions. The recommended use of HaloTag618 ligand for the NanoLuc-HaloTag BRET pair is versatile for ensemble experiments due to their well-separated emission bands. However, this system is not applicable for single-cell BRET imaging because of its low BRET efficiency and in turn weak acceptor signals. Here we explored the unprecedented potential of rhodamine based HaloTag ligands, containing azetidine rings, as BRET acceptors. Through a comprehensive evaluation of various commercial and Janelia Fluor HaloTag ligands for improved BRET efficiency and minimal donor signal bleed-through, we identified JF525 to be the best acceptor for microscopic BRET imaging. We successfully employed BRET imaging with JF525 to monitor the interaction of protein kinase A catalytic and regulatory subunit. Single-cell BRET imaging with HaloTag JF525 can henceforth open doors to comprehend and interpret molecular interactions.

Leigh Stoddart

University of Nottingham,

United Kingdom Abhijit De,

Tata Memorial Hospital, India

${ }^{*}$ Correspondence:

Hideaki Mizuno

hideaki.mizuno@kuleuven.be

Specialty section: This article was submitted to

Chemical Biology,

a section of the journal

Frontiers in Chemistry

Received: 14 October 2019 Accepted: 23 December 2019 Published: 14 January 2020

Citation:

Thirukkumaran OM, Wang C, Asouzu NJ, Fron E, Rocha S,

Hofkens J, Lavis LD and Mizuno $H$

(2020) Improved HaloTag Ligand Enables BRET Imaging With NanoLuc.

Front. Chem. 7:938.

doi: 10.3389/fchem.2019.00938
Keywords: NanoLuc, HaloTag, BRET imaging, Janelia Fluor dyes, PKA

\section{INTRODUCTION}

Förster resonance energy transfer (FRET) is a process of transferring excited-state energy from one chromophore (donor) to another (acceptor) via non-radiative dipole-dipole coupling. FRET has been a popular choice to detect interaction and conformational change of molecules since FRET depends on distance and relative orientation between the donor and acceptor. The development of a palette of genetically-encoded fluorescent proteins (FPs) has enabled the design of a variety of FRET-based biosensors to monitor intracellular phenomena (Sanford and Palmer, 2017). However, excitation of the donor FP by light illumination, an essential requisite for fluorescence imaging, might induce undesired system perturbation such as cell damage due to phototoxicity, photobleaching, and production of autofluorescence from intrinsic molecules.

Bioluminescence resonance energy transfer (BRET) is a category of FRET with the substitution of a fluorescent donor with a luminescent protein. Since bioluminescence is an intrinsic byproduct of an enzymatic reaction catalyzed by luciferases, BRET eliminates the need for an external excitation light. Employing the previous generation of luciferases (such as Firefly or Renilla luciferases) for imaging requires long exposure time even with a sensitive detection system due to very weak luminescence. Recently a bright luciferase, NanoLuc (England et al., 2016), which is small and emits blue luminescence has been engineered to be a good BRET donor (Machleidt et al., 2015; Hiblot et al., 2017). BRET sensors with NanoLuc and acceptor FP have been developed 
and applied for live cell imaging (Schaub et al., 2015; Hamer et al., 2017). Besides FPs, self-labeling protein tags such as HaloTag (Los et al., 2008) and SNAP/CLIP-tag (Keppler et al., 2003; Gautier et al., 2008) are alternative acceptors for BRET imaging. These genetically encodable enzyme tags catalyze a covalent bond formation with their fluorescently labeled cognate ligands. Amongst a series of membrane-permeable ligands for live cell assays, HaloTag NanoBRET 618 (Halo618) has been proposed as an appropriate BRET acceptor due to its wellseparated emission band that minimizes the impact of signal bleed-through from NanoLuc. The NanoLuc-Halo618 pair has been employed for ensemble BRET experiments (Machleidt et al., 2015; White et al., 2017). However, from a weak absolute acceptor signal employing Halo618, it is difficult to obtain information such as dynamics and heterogeneity from nonsynchronized cells via single-cell BRET imaging. The weak signal is due to the limited spectral overlap between NanoLuc emission and Halo618 absorption resulting in a lower BRET efficiency. In contrast, the superior spectral overlap of greenshifted acceptors are expected to improve BRET efficiency although the bleed-through of donor-signal into the acceptor window might become prominent. To enable live cell BRET imaging with NanoLuc, we evaluated rhodol and rhodamine based HaloTag ligands containing azetidine rings [Janelia Fluor 503 (JF503), Janelia Fluor 525 (JF525), Janelia Fluor 549 (JF549)], in comparison to oregon green (OG), tetramethylrhodamine (TMR) and Halo618, in the green-red spectral range for BRET efficiency, acceptor signal intensity and the impact of donor bleed-through.

\section{MATERIALS AND METHODS}

\section{Protein Expression and Purification}

E. coli JM109(DE3) was transformed with the respective bacterial expression vectors encoding either NanoLuc or Halo-NanoLuc. HaloTag was cloned to the C-terminus of NanoLuc with a spacer (SGGS). Transformed bacterial cells were grown, induced with IPTG for protein expression and later harvested to purify the expressed protein. The purified proteins were used for in vitro evaluation (details on cloning and protein purification can be found in Supplementary Material).

\section{Spectral Acquisitions in vitro \\ Fluorescence Spectra of HaloTag Ligands}

Synthesis of JF503, JF525, JF549, and spectroscopic measurements for HaloTag ligands (Supplementary Figure 2) were performed as described previously (Grimm et al., 2015, 2017). Absorption and fluorescence spectra (except for Halo618) were recorded with Cary Model 100 spectrometer (Varian) and Cary Eclipse fluorometer (Varian), respectively. The absorption spectrum of Halo618 was acquired with NanoDrop 2000 (Thermo Fischer Scientific). Quantum yields were determined using a Quantaurus-QY spectrometer (C11374, Hamamatsu) employing an integrating sphere to measure photons absorbed and emitted by a sample. Reported are average values from triplicates.

\section{Luminescence Spectra}

Halo-NanoLuc protein was incubated with HaloTag ligands (OG, JF503, JF525, JF549, TMR, and Halo618 $5 \mu \mathrm{M}$ ) at ambient temperature for $\sim 4 \mathrm{~h}$ to facilitate complete binding. Luminescence spectra for NanoLuc alone or Halo-NanoLuc labeled with the respective ligands were acquired with a final protein concentration of $4 \mathrm{nM}$ in PBS $+0.1 \%$ BSA in the presence of furimazine. Luminescence was collected within a few minutes after adding furimazine with a 30$\mathrm{mm}$ lens and sent to a spectrograph (Acton SpectroPro-300i monochromator/spectrograph). The dispersed luminescence from the spectrograph was detected with a sensitive liquid nitrogen cooled CCD camera (Princeton Instruments SPEC 10:100B/LNeXcelon). The acquisition time was set to $5 \mathrm{~s}$, the recorded spectra were averaged 10 times and background subtracted.

\section{BRET Imaging in Living Cells}

Imaging experiments were all performed on transfected cells (protocol in Supplementary Material), within a few minutes after furimazine (1:30 dilution) addition, on an inverted microscope IX83 (Olympus, Tokyo) with an APON 60XOTIRF $(\mathrm{NA}=1.49)$ objective lens (Olympus). Luminescence was split into donor and acceptor windows with a dichroic mirror DM509 (Semrock, NY; OG, JM503, and JM525) or DM555 (Semrock; JF549, TMR, and Halo618). Donor and acceptor images were acquired simultaneously with two EM-CCD cameras (ImagEM C9100-13, Hamamatsu Photonics, Hamamatsu, Japan). The images for Halo-NanoLuc were acquired with $2 \mathrm{x}$ binning $(0.55 \mu \mathrm{M}$ per pixel), $1200 \mathrm{EM}$ gain and $0.75 \mathrm{~s}$ exposure time (averaged 2x). To observe PKA dissociation events, time traces were recorded for $7 \mathrm{~min}$ with $2 \mathrm{x}$ binning, $297 \mathrm{EM}$ gain and $1 \mathrm{~s}$ exposure time (averaged $2 \mathrm{x}$ ). After $\sim 3 \mathrm{~min}$, cells were stimulated by adding $10 \mu \mathrm{M}$ forskolin through a winged infusion set $-22 \mathrm{G} \times 3 / 4$ " (Terumo, Tokyo, Japan). Acceptor/Donor emission ratios $\left(\mathrm{R}_{\mathrm{A} / \mathrm{D}}\right)$ were calculated from regions of interest (ROI) in individual cells using Fiji (Schindelin et al., 2012). Background from each window was calculated from an ROI without any cells. The background-corrected values were used for further analyses. The different HaloTag ligands were imaged with the same imaging parameters except for the choice of dichroic mirrors as indicated.

\section{RESULTS}

\section{Green-Shifted HaloTag Ligands Show Better BRET Efficiency}

For the in vitro evaluation, we acquired the emission spectrum of recombinant NanoLuc with substrate furimazine and the absorption spectra of different HaloTag ligands: OG, JF503, (Grimm et al., 2017) JF525, (Grimm et al., 2017) JF549, (Grimm et al., 2015), TMR and Halo618 (Figure 1A). Since the efficiency of energy transfer linearly correlates to the overlap integral $(J)$, $J$ was calculated using the acquired spectra (Table 1). JF503 had the largest $J\left(1.8 \times 10^{13} \mathrm{M}^{-1} \mathrm{~cm}^{3}\right)$ followed by OG and JF525 (83\% of JF503), JF549 (72\% of JF503), and TMR (56\% of JF503). For Halo618, $J$ could not be calculated since its extinction 


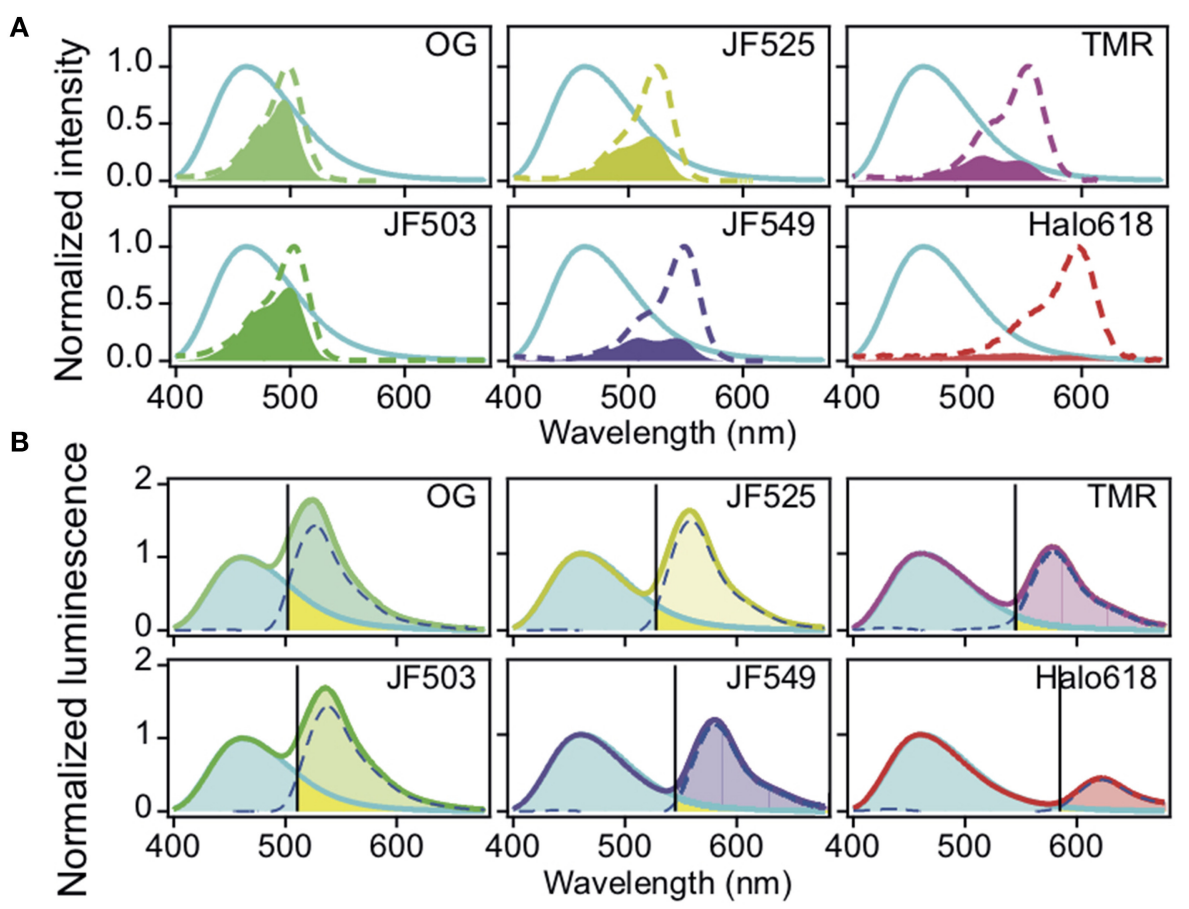

FIGURE 1 | Spectra of NanoLuc and HaloTag ligands. (A) Normalized absorption spectra of HaloTag ligands. The spectra of the different ligands are in dotted lines (OG, bluish green, JF503, yellowish green, JM525, yellow, JF549, violet, TMR, purple, Halo618, red) and overlaid with the normalized luminescence spectrum of NanoLuc (cyan solid line). The filled region indicates the overlap area (the products of two spectra). (B) Luminescence spectra of Halo-NanoLuc conjugated with Halo-ligands. Spectra were normalized with the donor peak at $460 \mathrm{~nm}$. Dotted blue lines indicate the acceptor spectrum calculated by subtracting the spectrum for only NanoLuc (cyan) from that with the ligand. The wavelength where the acceptor spectrum intersects the NanoLuc spectrum $\left(\mathrm{S}_{\mathrm{D}: \mathrm{A}}\right)$ is denoted with a black line. $\mathrm{R}_{\mathrm{b}}$ is the ratio of yellow area over sum of yellow and cyan areas, whereas $F_{D}$ is the ratio of yellow area over sum of the yellow area and the area colored with the color code of respective ligands.

TABLE 1 | Evaluation of HaloTag ligands as BRET acceptors of NanoLuc in vitro ${ }^{\text {. }}$

\begin{tabular}{|c|c|c|c|c|c|c|c|c|c|}
\hline Ligand & $\begin{array}{c}\text { Abs }_{\max } \\
(\mathrm{nm})\end{array}$ & $\left(\mathrm{M}^{-1} \mathrm{~cm}^{-1}\right)$ & $\begin{array}{c}\mathrm{Em}_{\max } \\
(\mathrm{nm})\end{array}$ & $\Phi$ & $\begin{array}{c}J \\
\left(\mathrm{M}^{-1} \mathrm{~cm}^{3}\right)\end{array}$ & $\mathrm{I}_{\mathrm{A}}$ & $\begin{array}{l}\mathrm{S}_{\mathrm{D}: A} \\
(\mathrm{~nm})\end{array}$ & $\begin{array}{l}R_{b t} \\
(\%)\end{array}$ & $\begin{array}{l}F_{D} \\
(\%)\end{array}$ \\
\hline OG & 496 & $8.3 \times 10^{4}$ & 526 & 0.88 & $1.5 \times 10^{13}$ & 86.4 & 502 & 25.5 & 22.1 \\
\hline JF503 & 503 & $8.3 \times 10^{4}$ & 529 & 0.87 & $1.8 \times 10^{13}$ & 90.1 & 511 & 19.9 & 17.2 \\
\hline JF525 & 525 & $9.4 \times 10^{4}$ & 549 & 0.91 & $1.5 \times 10^{13}$ & 82.5 & 528 & 12.1 & 11.9 \\
\hline JF549 & 549 & $1.0 \times 10^{5}$ & 571 & 0.88 & $1.3 \times 10^{13}$ & 63.7 & 547 & 6.9 & 8.8 \\
\hline TMR & 554 & $7.8 \times 10^{4}$ & 572 & 0.41 & $1.0 \times 10^{13}$ & 65.7 & 545 & 7.4 & 9.8 \\
\hline Halo618 & 595 & $N D^{b}$ & $621^{c}$ & $N D^{b}$ & $N D^{b}$ & 22.7 & 585 & 2.3 & 8.2 \\
\hline
\end{tabular}

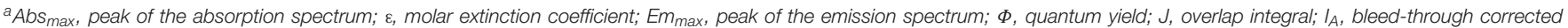
signal intensity from the acceptor; $S_{D: A}$, the split point between donor and acceptor windows; $R_{b t}$, ratio of NanoLuc signal in the acceptor window to the entire NanoLuc signal; $F_{D}$, fraction of donor signal in the acceptor window relative to the entire acceptor signal. Refer Supplementary Material for calculation of $J$, $R_{b t}$, and $F_{D}$.

${ }^{b}$ Not determinable due to lack of information on material synthesis.

${ }^{c}$ Data from Promega.

coefficient was not available. Assuming Halo618 has an extinction coefficient comparable to the other HaloTag ligands, a $J$ smaller than TMR is expected from the overlapped area calculated with the normalized spectra.

To experimentally evaluate the BRET efficiency, we acquired the emission spectra of recombinant NanoLuc-HaloTag tandem protein (Halo-NanoLuc) conjugated with the HaloTag ligands (Figure 1B). After normalizing the spectra with the maximum emission of NanoLuc (at $460 \mathrm{~nm}$ ), signal intensity derived from the acceptor molecule was calculated by subtracting integral of the spectrum for only NanoLuc from that of Halo-NanoLuc conjugated with the ligand $\left(\mathrm{I}_{\mathrm{A}}\right)$ (Table 1 ). Since the spectra were acquired in the dark, energy exciting the acceptor was provided exclusively through BRET, and therefore $\mathrm{I}_{\mathrm{A}}$ reflects the BRET efficiency as well as the fluorescence quantum yield of the acceptor $(\Phi)$. As expected from calculated $J$ and $\Phi$, JF503 
showed the highest BRET efficiency $\left(I_{\mathrm{A}}=90.1\right)$ followed by OG (96\% of JF503) and JF525 (92\% of JF503). $\mathrm{I}_{\mathrm{A}}$ values of JF549 and TMR were about $70 \%$ of JF503 whereas Halo618 was only $25 \%$ of JF503. Therefore, we concluded that in terms of BRET efficiency green-shifted ligands performed as better acceptors, which was in accordance with previous reports on non-JF dyes (Machleidt et al., 2015; Hiblot et al., 2017).

\section{Quantifying Donor Signal Bleed-Through to Identify Best Acceptor}

Another crucial factor we evaluated is the donor signal bleed-through. To quantify bleed-through, we determined the wavelength $\left(\mathrm{S}_{\mathrm{D}: \mathrm{A}}\right)$ at the intersection of NanoLuc and the acceptor spectra (Figure 1B). Based on $S_{D: A}$ value, we calculated the ratio of NanoLuc signal in acceptor window relative to entire NanoLuc signal $\left(\mathrm{R}_{\mathrm{bt}}\right)$, and the fraction of signal derived from donor molecules relative to entire signal in the acceptor window $\left(F_{D}\right) . R_{b t}$ gives the degree of bleed-through whereas $\mathrm{F}_{\mathrm{D}}$ indicates the impact of this bleed-through in the acceptor window. Bleed-through was insignificant for Halo618 $\left(\mathrm{R}_{\mathrm{bt}}=\right.$ $2.3 \%$ ), and it became more prominent for ligands with emission at shorter wavelengths. The $\mathrm{R}_{\mathrm{bt}}$ values of the ligands in the orange range (JF549, TMR) were about three-fold higher than Halo618. Nevertheless, the impact of bleed through $\left(\mathrm{F}_{\mathrm{D}}\right)$ was similar to Halo618 because of a three-fold better acceptor signal $\left(\mathrm{I}_{\mathrm{A}}\right)$ (Table 1). On the other hand, ligands in green range (JF525, JF503, and OG) had $R_{b t}$ values 5-11-fold higher than Halo618. In spite of such large $\mathrm{R}_{\mathrm{bt}}$ values, the $\mathrm{F}_{\mathrm{D}}$ values were only 1.5-, 2.1-, 2.7-fold of Halo618 due to brighter acceptor signal $\left(I_{\mathrm{A}}\right)$. From this result, we concluded that the impact of bleed-through was not significant compared to the ratio of bleed-through for green-shifted ligands.

\section{JF525 Performs as an apt Acceptor of NanoLuc}

We next evaluated the capability of these different HaloTag ligands for live cell BRET imaging. Chinese hamster ovary (CHO-K1) cells expressing Halo-NanoLuc were labeled with the respective HaloTag ligands and then imaged with a microscope (refer section BRET Imaging in Living Cells for details). The luminescent signals were split into a donor and acceptor window using a dichroic mirror DM509 for the green-shifted ligands (OG, JF503, and JF525) and with DM555 for orange-red ligands (JF549, TMR, and Halo618). Luminescence intensity varies cell by cell depending on the expression level of protein and consumption of furimazine. Therefore, the BRET efficiency was evaluated by comparing the acceptor signal to the donor signal. Bright luminescence signals were observed in a lot of OG-, JF503-, and JF525-loaded cells in both donor and acceptor windows with $0.75 \mathrm{~s}$ exposure time, whereas only donor signal was visible in unloaded cells (Figure 2A). In JF549-, TMR-, and Halo618-loaded cells, signals in the acceptor window were barely visible even in cells whose signal in donor window was comparable to OG-, JF503-, and JF525loaded cells. For quantitative evaluation, signal intensities from acceptor and donor windows were measured for 70 cells or more, and their ratio $\left(\mathrm{R}_{\mathrm{A} / \mathrm{D}}\right)$ was calculated by performing linear regression analyses (Figures 2B-D). JF525-loaded cells gave the best acceptor signal with the $\mathrm{R}_{\mathrm{A} / \mathrm{D}}$ value of $0.82 \pm 0.01$ (Mean \pm SEM). It was 6.8 -fold larger than the bleed-through signal measured in cells without ligands using the same experimental $\operatorname{setup}\left(\mathrm{R}_{\mathrm{A} / \mathrm{D}}=0.12 \pm 0.00\right)$. JF503 $\left(\mathrm{R}_{\mathrm{A} / \mathrm{D}}=0.58 \pm 0.01\right)$ and $\mathrm{OG}$ $\left(\mathrm{R}_{\mathrm{A} / \mathrm{D}}=0.45 \pm 0.00\right)$-loaded cells showed signals that were 4.8 and 3.8-fold more than bleed-through. For JF549-, TMR-, and Halo618-loaded cells, the bleed-through was lower $\left(\mathrm{R}_{\mathrm{A} / \mathrm{D}}=0.02\right.$ $\pm 0.00)$ as a result of using DM555 instead of DM509, but the acceptor signal was remarkably weaker $\left(\mathrm{R}_{\mathrm{A} / \mathrm{D}}=0.36 \pm 0.00,0.21\right.$ \pm 0.00 , and $0.24 \pm 0.00$, respectively) than JF525-loaded cells. We concluded that JF525 was the best acceptor for BRET imaging with NanoLuc.

\section{Monitoring PKA Activation Through BRET Imaging}

Resonance energy transfer systems are frequently employed to monitor protein interactions. Therefore, we evaluated the suitability of BRET imaging with NanoLuc and JF525 for this application. We observed the interaction of regulatory and catalytic subunits of protein kinase A (PRKAR2A and PRKACA, respectively), as these subunits exist together as tetramers under resting conditions (Figure $\mathbf{3 A}$ ) and undergo a rapid dissociation in response to increase in cAMP levels (Figure 3B) (Taylor et al., 1990; Knighton et al., 1991). We labeled the N-terminus of regulatory (RS)- and C-terminus of catalytic (CS)-subunits with NanoLuc (NL) and HaloTag (HT), respectively (NL-RS, CS-HT). Both the subunits were co-expressed in NIH3T3 cells, labeled with HaloTag ligands and were imaged in the presence of furimazine. Signals were visible in both donor and acceptor windows for cells loaded with JF525 (Figure 3C). From the intensity time trace of the donor and acceptor signal from multiple cells $(n=8)$, the signal to noise ratios (SNRs) for JF525 were determined to be $249.7 \pm 18$ (donor) and $165.5 \pm 14.2$ (acceptor) (Figures 3D,E and Supplementary Figure 1). On the contrary, only a faint acceptor signal was observed with Halo618 even for cells showing similar donor intensities as JF525. The difference in donor SNRs was insignificant between JF525 and Halo618 (191.9 $\pm 13.4 ; p>0.05)$ while the acceptor SNR for Halo618 (100.9 \pm 8.9 ) was significantly lower $(p<0.005)$. The dissociation of PKA subunits was evident through a decrease in $\mathrm{R}_{\mathrm{A} / \mathrm{D}}$ upon forskolin, an agonist for adenylyl cyclase to increase cAMP level, addition for cells loaded with JF525 and Halo618 (Figure 3F). However, the response $\left(\Delta \mathrm{R}_{\mathrm{A} / \mathrm{D}}\right)$ for JF525 was 0.012 \pm 0.006 (median $\pm \mathrm{SD}$ ), which is twice as much as $\Delta \mathrm{R}_{\mathrm{A} / \mathrm{D}}$ for Halo618 (0.007 $\pm 0.002 ; p<0.05)$ (Figure 3G). JF525 evidently showed much brighter acceptor signals suitable to monitor protein interactions by BRET imaging with single-cell resolution.

\section{DISCUSSION}

Self-labeling protein tags such as HaloTag provide a sophisticated platform to evaluate the acceptors in an individual BRET system due to the availability of versatile bright, photostable, and cell permeable ligands. Usage of HaloTag618 as a recommended acceptor for NanoLuc minimizes donor signal bleed-through in the acceptor window (Machleidt et al., 2015). However, a very low energy transfer efficiency yields poor acceptor 
A
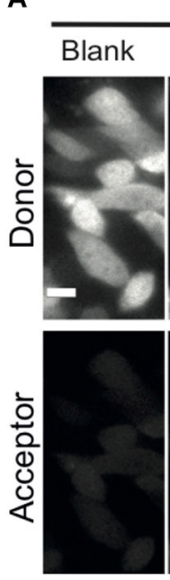

DM509
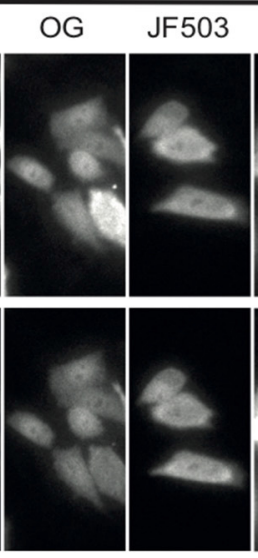

DM555

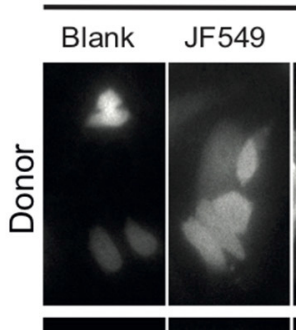

JF503 JF525
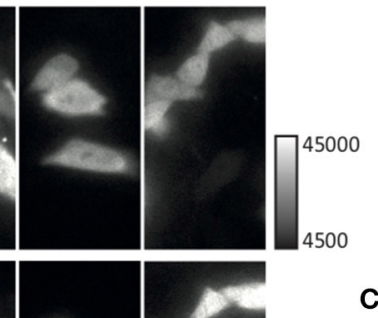

C

B
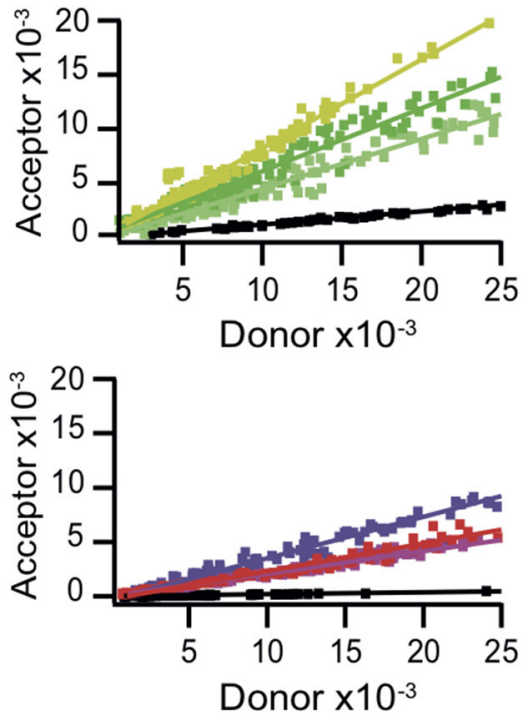

D

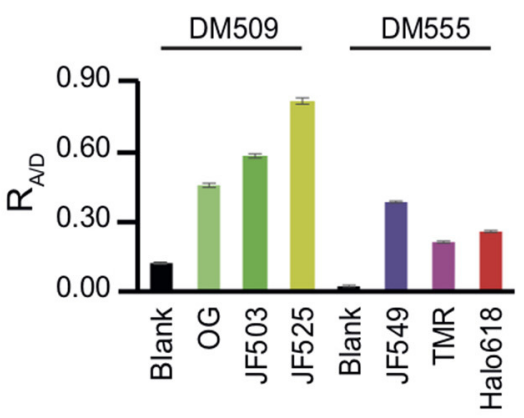

FIGURE 2 | BRET imaging in living cells. (A) Images of CHO-K1 cells expressing Halo-NanoLuc. Images were acquired after loading of respective ligands (OG, JF503, JF525, JM549, TMR, and Halo618) or in the absence of ligands (blank), using a dichroic mirror indicated to separate donor (upper) and acceptor (lower) windows. All images were obtained with identical acquisition settings (APON 60XOTIRF, NA = 1.49, exposure time $=0.75 \mathrm{~s}$ ). Scale bar $=10 \mu \mathrm{m}$. (B,C) Linear regression analyses of the acceptor vs. donor signal intensities for green-shifted (B) or orange-red ligands (C). The color codes of ligand-loaded cells are same as in Figure 1. Cells in the absence of ligands (blank) are shown in black. (D) Mean $\mathrm{R}_{\mathrm{A} / \mathrm{D}} \pm \mathrm{SEM}$ calculated from the cells labeled with different ligands. $n \geq 70$.

signal largely hampering its application in the direction of cellular imaging. Employing ligands in the green spectral region, such as Alexa 488, have been reported to show higher BRET efficiency (Machleidt et al., 2015; Hiblot et al., 2017). Since higher bleed-through was expected with green ligands, they were not popularized for ensemble experiments. Conversely, higher absolute acceptor signals are appreciated for BRET imaging. Therefore, we performed a systematic evaluation of a series of HaloTag ligands to identify the best BRET acceptors of NanoLuc for live cell imaging. Brightness comparison amongst the ligands in the green-yellow spectral range (OG, JG503, and JF525) showed JF525 to be better because of a higher extinction coefficient and quantum yield although the overlap integral was lower than JF503. On the other hand, a comprehensive analysis indicated bleed-through to be 1.5 -fold lower than OG. With an extensive evaluation of the bleed-through relative to BRET efficiency, we concluded that JF525 was the best in vitro amongst the other ligands. Endorsing the in vitro results, from cellular
BRET imaging it was evident that JF525 showed the highest BRET ratio.

We translated the application of this BRET system with JF525 to monitor protein-protein interaction of the labeled catalytic and regulatory subunits of PKA. Under resting conditions, we observed a basal BRET ratio which showed the interaction of these subunits. Upon stimulation with forskolin, a drop in BRET ratio was detected due to the dissociation of regulatory and catalytic subunits which enabled us to monitor PKA activation. The observed contrast upon stimulation allowed us to monitor dynamic protein-interaction within the sensor system. Apart from monitoring protein-protein interactions, the application of this system could be extended to image the interaction of proteins with small molecules that can be labeled with JF dyes. Moreover, BRET imaging could be interesting to observe interactions on light sensitive cells, avoid phototoxicity and cellular autofluorescence associated with excitation. 

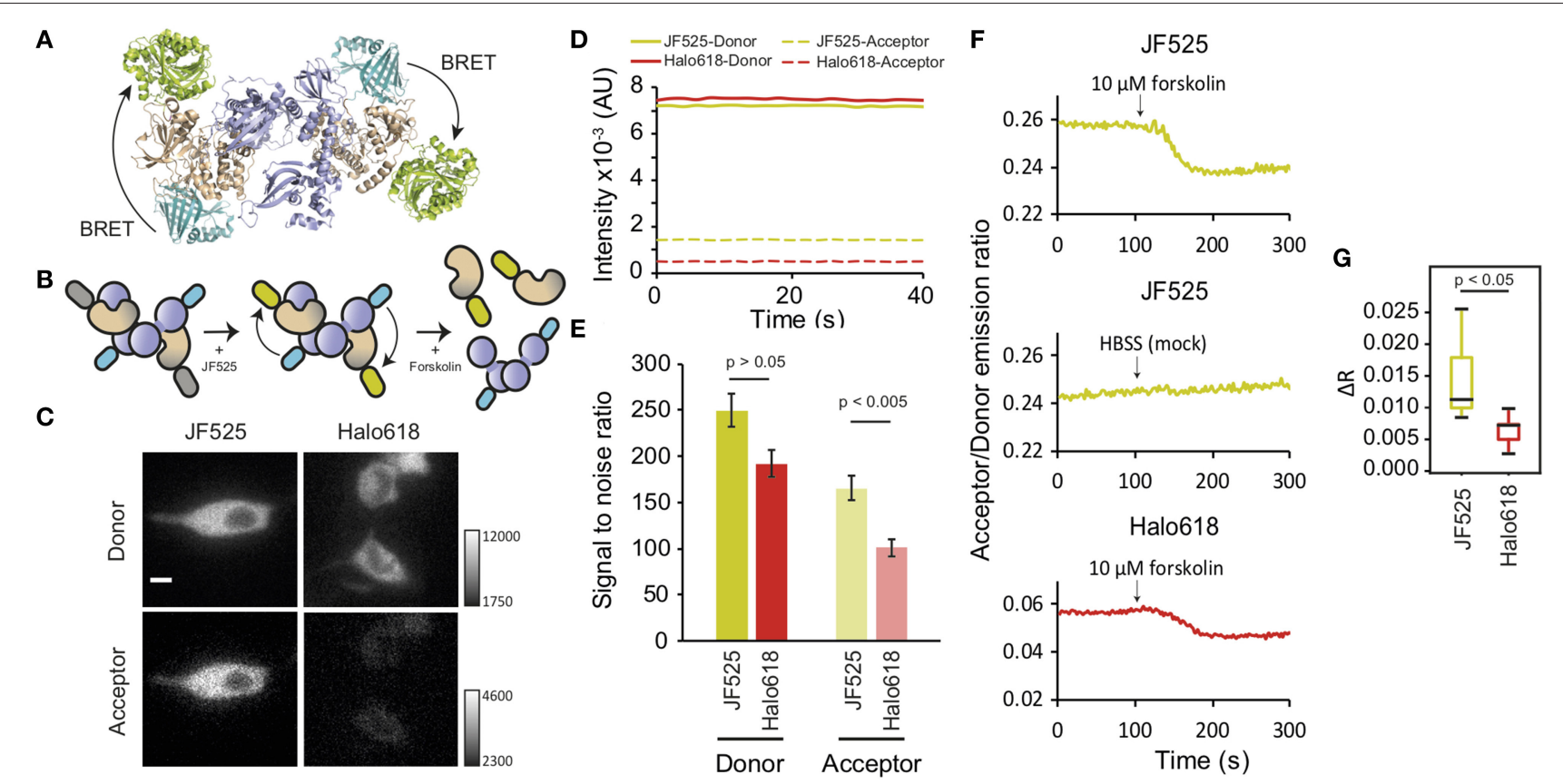

FIGURE 3 | Dissociation of PKA subunits observed by BRET. (A) Interaction of NL-RS and CS-HT modeled with the crystal structures (PDB files: 2QBVS, 2F7E, 5IBO, 5Y2Y). The regulatory subunit is shown in purple, catalytic subunit in wheat, NanoLuc in cyan, and HaloTag in greenish-yellow. (B) Schematic explaining the decrease in BRET upon dissociation of PKA subunits. The tetrameric structure of tagged NL-RS and CS-HT under resting condition (left) is labeled with HaloTag ligands (middle) and stimulated with forskolin to dissociate the interaction of RS and CS (right). (C) Luminescence images of NIH3T3 cells co-expressing NL-RS and CS-HT. Images were acquired after loading respective HaloTag ligands (JF525 and Halo618). All images were acquired with identical camera settings. Scale bar = $10 \mu \mathrm{m}$. (D) Representative intensity traces of donor and acceptor signals and (E) Mean SNR \pm SEM for cells labeled with either JF525 or Halo618. (F) Time traces of acceptor/donor emission ratios showing dissociation of PKA subunits NL-RS and CS-HT upon forskolin stimulation (indicated by black arrow). (G) Box plot showing $\Delta R_{A / D}$ values for cells labeled with respective HaloTag ligands. $n=8$.

In addition to the photochemical properties, cellular permeability and labeling efficiency are important for live cell imaging. Since the Oregon Green fluorophore $\left(2^{\prime}, 7^{\prime}-\right.$ difluorofluorescein) has two $\mathrm{OH}$ groups at the 3,9' positions of the xanthene ring (Sun et al., 1997), the fluorophore itself is hydrophilic and cell impermeable. The OG HaloTag ligand is permeabilzed by diacetylating these two $\mathrm{OH}$ groups (Supplementary Figure 2). However, the ester bonds are chemically less stable in aqueous solution (Lavis et al., 2011) and its deacetylation decreases the propensity of OG to be cell permeable. Throughout our experiments, fresh stocks of OG were used to avoid this bias. In the case of JF ligands, the presence of azetidine group at the positions $3,9^{\prime}$ of the xanthene ring makes them hydrophobic, facilitating cell permeability. Hence, the stability of JF ligands is not expected to influence the BRET efficiency.

In order to achieve complete labeling in living cells, binding of OG, TMR, and Halo618 was done according to the protocol recommended by Promega. For the JF ligands, we established a protocol based on the reported time course of HistoneH2B labeling with JF HaloTag ligands (Grimm et al., 2017). Under these conditions, a complete labeling is expected to identify the best ligands. Ligands unable to achieve this labeling efficiency introduce underestimation of their BRET efficiencies and such ligands are in any case not appropriate as BRET acceptors.
In conclusion, we have demonstrated that the brightness, cellular permeability, labeling efficiency, photostability of HaloTag ligands, and impact of bleed-through signals relative to acceptor signals are crucial factors for BRET efficiency. Analyses in vitro revealed that green-shifted ligands outperformed the red-shifted ligands in terms of better BRET efficiency without marked interference from bleed-through of donor signal. Among the green-shifted ligands, JF525 was identified as the best acceptor for BRET imaging. Bright acceptor images could be acquired from single-cells employing JF525 with an exposure time of $<1 \mathrm{~s}$. BRET imaging with JF525 serves as an improved platform to investigate dynamic molecular interactions in single-cells.

\section{DATA AVAILABILITY STATEMENT}

All datasets from this study are included in the manuscript and the Supplementary Files. The raw data files would be available upon request to the corresponding author.

\section{AUTHOR CONTRIBUTIONS}

OT and HM conceptualized, designed the experiments and wrote the manuscript. OT, HM, EF, CW, and NA acquired the absorption spectra and the luminescence spectra, and 
performed statistical analyses. LL synthesized and carried out the in vitro evaluation of Janelia Fluor (JF) ligands. JH and SR imparted microscopic knowledge and adapted the microscope for bioluminescence imaging. JH, SR, LL, and HM improvised, revised, and approved the submitted version of manuscript.

\section{FUNDING}

This work was supported by the KU Leuven Category 1 research grant from KU Leuven (C14/16/053). OT was

\section{REFERENCES}

England, C. G., Ehlerding, E. B., and Cai, W. (2016). NanoLuc: a small luciferase is brightening up the field of bioluminescence. Bioconjug. Chem. 27, 1175-1187. doi: 10.1021/acs.bioconjchem.6b00112

Gautier, A., Juillerat, A., Heinis, C., Corrêa, I. R., Kindermann, M., Beaufils, F., et al. (2008). An engineered protein tag for multiprotein labeling in living cells. Chem. Biol. 15, 128-136. doi: 10.1016/j.chembiol.2008.01.007

Grimm, J. B., English, B. P., Chen, J., Slaughter, J. P., Zhang, Z., Revyakin, A., et al. (2015). A general method to improve fluorophores for live-cell and single-molecule microscopy. Nat. Methods. 12, 244-250. doi: 10.1038/ nmeth.3256

Grimm, J. B., Muthusamy, A. K., Liang, Y., Brown, T. A., Lemon, W. C., Patel, R., et al. (2017). A general method to fine-tune fluorophores for live-cell and in vivo imaging. Nat. Methods. 14, 987-994. doi: 10.1038/nmeth.4403

Hamer, A., Den Dierickx, P., Arts, R., De Vries, J. S. P. M., Brunsveld, L., and Merkx, M. (2017). Bright bioluminescent BRET sensor proteins for measuring intracellular caspase activity. ACS Sensors 2, 729-734. doi: 10.1021/acssensors.7b00239

Hiblot, J., Yu, Q., Sabbadini, M. D. B., Reymond, L., Xue, L., Schena, A., et al. (2017). Luciferases with tunable emission wavelengths. Angew. Chem. 7, 14556-14560. doi: 10.1002/anie.201708277

Keppler, A., Gendreizig, S., Gronemeyer, T., Pick, H., Vogel, H., and Johnsson, K. (2003). A general method for the covalent labeling of fusion proteins with small molecules in vivo. Nat. Biotechnol. 21, 86-89. doi: 10.1038/ nbt765

Knighton, D. R., Zheng, J. H., Ten Eyck, L. F., Xuong, N. H., Taylor, S. S., and Sowadski, J. M. (1991). Structure of a peptide inhibitor bound to the catalytic subunit of cyclic adenosine monophosphate-dependent protein kinase. Science 253, 414-420. doi: 10.1126/science.1862343

Lavis, L. D., Chao, T. Y., and Raines, R. T. (2011). Synthesis and utility of fluorogenic acetoxymethyl ethers. Chem. Sci. 2, 521-530. doi: 10.1039/C0SC00466A

Los, G. V, Encell, L. P., Mcdougall, M. G., Hartzell, D. D., Karassina, N., Zimprich, C., et al. (2008). HaloTag: a novel protein labeling technology for cell imaging and protein analysis. ACS Chem. Biol. 3, 373-382. doi: 10.1021/ cb800025k supported by an Aspirant $\mathrm{PhD}$ grant from the Research Foundation-Flanders (FWO-1147619N). This publication was made possible through funding support of the KU Leuven Fund for Fair Open Access.

\section{SUPPLEMENTARY MATERIAL}

The Supplementary Material for this article can be found online at: https://www.frontiersin.org/articles/10.3389/fchem.2019. 00938/full\#supplementary-material

Machleidt, T., Woodroofe, C. C., Schwinn, M. K., Méndez, J., Robers, M. B., Zimmerman, K., et al. (2015). NanoBRET-a novel BRET platform for the analysis of protein-protein interactions. ACS Chem. Biol. 10, 1797-1804. doi: 10.1021/acschembio.5b00143

Sanford, L., and Palmer, A. (2017). Recent advances in development of genetically encoded fluorescent sensors. Meth. Enzymol. 589, 1-49. doi: 10.1016/bs.mie.2017.01.019

Schaub, F. X., Reza, M. S., Flaveny, C. A., Li, W., Musicant, A. M., Hoxha, S., et al. (2015). Fluorophore-NanoLuc BRET reporters enable sensitive in vivo optical imaging and flow cytometry for monitoring tumorigenesis. Cancer Res. 75, 5023-5033. doi: 10.1158/0008-5472.CAN-14-3538

Schindelin, J., Arganda-Carreras, I., Frise, E., Kaynig, V., Longair, M., Pietzsch, T., et al. (2012). Fiji: an open-source platform for biological-image analysis. Nature methods 9, 676-682. doi: 10.1038/nmeth.2019

Sun, W. C., Gee, K. R., Klaubert, D. H., and Haugland, R. P. (1997). Synthesis of fluorinated fluoresceins. J. Org. Chem. 62, 6469-6475. doi: 10.1021/jo97 06178

Taylor, S. S., Buechler, J. A., and Yonemoto, W. (1990). cAMP-dependent protein kinase: Framework for a diverse family of regulatory enzymes. Annu. Rev. Biochem. 59, 971-1005. doi: 10.1146/annurev.bi.59.070190. 004543

White, C. W., Vanyai, H. K., See, H. B., Johnstone, E. K. M., and Pfleger, K. D. G. (2017). Using nanoBRET and CRISPR/Cas9 to monitor proximity to a genome-edited protein in real-time. Sci. Rep. 7:3187. doi: 10.1038/s41598-01703486-2

Conflict of Interest: The authors declare that the research was conducted in the absence of any commercial or financial relationships that could be construed as a potential conflict of interest.

Copyright (C) 2020 Thirukkumaran, Wang, Asouzu, Fron, Rocha, Hofkens, Lavis and Mizuno. This is an open-access article distributed under the terms of the Creative Commons Attribution License (CC BY). The use, distribution or reproduction in other forums is permitted, provided the original author(s) and the copyright owner(s) are credited and that the original publication in this journal is cited, in accordance with accepted academic practice. No use, distribution or reproduction is permitted which does not comply with these terms. 